\title{
Comparative evaluation of salivary alpha amylase level for assessment of stress during third molar surgery with and without piano music and co-relation with pain catastrophizing scale: an in vivo study
}

\author{
Vibha Vaswani, Sonal Shah, Manne Lakshmipriyanka, Pushkar Waknis, Deeisha Gupta, Kunal Jain \\ Department of Oral \& Maxillofacial Surgery, Dr. D. Y. Patil Vidyapeeth, Pune, India
}

\begin{abstract}
J Korean Assoc Oral Maxillofac Surg 2020;46:235-239)
Objectives: Preoperative nervousness and anxiety are frequently encountered by individuals who undergo extractions of impacted wisdom teeth. The aim of the present study is to evaluate salivary alpha amylase (sAA) level in patients for assessment of stress during third molar surgery while listening to piano music and to determine its co-relation with pain catastrophizing scale (PCS).

Materials and Methods: Seven patients (four males and three females) indicated for surgical extraction of bilaterally impacted mandibular third molars were included. Pre-surgical patient assessments were completed, and three samples of saliva were collected during surgery—one at baseline, one 30 minutes after commencement of surgery, and one after suturing. Assessment was performed on both sides separately with and without piano music, and the samples were assessed for sAA level and correlated with the patient's self-reported PCS.

Results: Statistically significant results were obtained in patients who underwent surgical extraction while listening to piano music $(P=0.046)$. The correlation of sAA level with PCS was not significant.

Conclusion: Music demonstrated a beneficial effect on lowering the levels of stress and anxiety that a patient exhibits during any surgical procedure, and sAA can be a useful biomarker for similar assessments.
\end{abstract}

Key words: Molar, Third, Stress, Biomarker, Saliva

[paper submitted 2019. 12. 23 / revised 1st 2020. 3. 9, 2nd 2020. 4. 2 / accepted 2020. 4. 3]

\section{Introduction}

Preoperative nervousness and anxiety are frequently encountered by individuals who undergo extraction of impacted wisdom teeth, one of the most common procedures performed in oral surgery. Individuals present with a variety of psychological and/or physiological responses to stress considering patient underlying personality traits ${ }^{1}$. More fear or distress before the surgical procedure can be associated with a delayed and complex recovery postoperatively. Anxiety hampers recovery through both physiological and psycho-

\section{Vibha Vaswani}

Department of Oral \& Maxillofacial Surgery, Dr. D. Y. Patil Vidyapeeth, Pimpri, Pune 411018, India

TEL: +91-2027805600 FAX: +91-2027423427

E-mail:vibha0340@gmail.com

ORCID: https://orcid.org/0000-0002-7504-7271

(c) This is an open-access article distributed under the terms of the Creative Commons Attribution Non-Commercial License (http://creativecommons.org/ licenses/by-nc/4.0/), which permits unrestricted non-commercial use, distribution, and reproduction in any medium, provided the original work is properly cited.

Copyright (C) 2020 The Korean Association of Oral and Maxillofacial Surgeons. All rights reserved. logical means ${ }^{2}$.

Pain severity, pain-related disability, and psychological adjustment are clinical factors that determine how an individual copes with pain. Catastrophizing in terms of both evaluation and coping is a negative, emotional, and empiric response to pain and is associated with elements such as exaggeration, helplessness, and rumination ${ }^{3,4}$. This predicts higher pain ratings in the context of dental procedures ${ }^{5}$.

Since a patient's inclination toward noxious stimuli and stressful experiences greatly hampers recovery from postoperative pain and discomfort, a systematic evaluation of this kind of psychological stress would enable the surgeon to have a more dynamic approach for management of pain, resulting in a better surgical experience for the patient ${ }^{1}$. As mentioned earlier, since expression of stress and anxiety relies on the individual's personality, assessment cannot be completely based on subjective grounds. In the past, researchers in the field of psychoneuroendocrinology have concentrated on methods to quantify the biological changes related to stress responses with continuous development of biomarkers for as- 
sessments.

Blood is the most widely used biofluid for evaluation of biomarkers, but it does not accurately assess the stress biomarker profile due to the known discomfort a patient experiences during procurement of blood samples. Saliva, on the other hand, is readily obtainable, can be procured with ease, and is a source of stress biomarkers like cortisol, salivary alpha amylase (sAA), and chromogranin-A. Among these, sAA has appeared as a prospective substitute marker of autonomous nervous system activity, suggesting that it may be a specific marker of the adrenergic system that is generated by psychological distress ${ }^{1,6-8}$.

Expression of sAA for assessment of distress depends on the advent of technologies that can yield quick and accurate reporting of sAA level. A portable, colorimetric, sAA biosensor, Cocoro meter, is used along with disposable strips (Cocoro meter chips) to measure the level of sAA ${ }^{9}$. Psychological effects including induction and modification of moods and emotions can be brought about by music due to its anxiolytic effects. Therefore, music is being combined with other medical therapies to help relieve perioperative surgery-related stress and anxiety ${ }^{10}$.

The present study is designed to establish the correlation between level of sAA and self-reported anxiety and stress through the pain catastrophizing scale (PCS) during surgical removal of wisdom teeth.

\section{Materials and Methods}

The present study was conducted after obtaining approval from the Institutional Ethics Committee of Dr. D. Y. Patil Dental College \& Hospital, Pune (DYPDCH/ IEC/1262/14118).

This was a retrospective study of 14 cases from seven patients who underwent surgical extraction of bilateral impacted mandibular third molars between 2017 and 2019 at the Department of Oral \& Maxillofacial Surgery of Dr. D. Y. Patil Dental College \& Hospital, Dr. D. Y. Patil Vidyapeeth.

The mean age of the patients was 29.85 years with an age range of 23 to 48 years. Four males and three females participated in the study.

Inclusion criteria were patients above 18 years of age who presented with impacted mandibular third molars that were indicated for surgical extraction and who voluntarily participated in the study. Patients who were on anti-anxiety medications were excluded.

Preoperative assessment involving a comprehensive case history ruling out the presence of any systemic comorbidities and a detailed history of anti-anxiety medications was obtained from all the participants. Routine investigations of hemoglobin, bleeding, and clotting times were assessed for all patients, who were subsequently scheduled for surgery.

\section{Treatment protocol}

Written informed consent was obtained from all participants after a detailed case history and clinical examination. Depending on whether the surgical extraction was to be performed with or without piano music and involved left or right side, patients were selected respectively. Three samples of saliva were collected using the Cocoro sAA biosensor (Nipro, Osaka, Japan).(Fig. 1)

On the first visit on the day of surgery, the patient was informed about the aim of the study and the method of saliva collection and was asked to fill out the PCS (Michael JL Sullivan, PhD Departments of Psychology, Medicine, and Neurology School of Physical and Occupational Therapy McGill University, Montreal, QC, Canada). The patient was asked to orally rinse with normal saline, and a baseline sample of preoperative saliva was collected, after which the patient was subjected to pre-recorded classical piano music.

While the music was playing, 30 minutes after commencement of surgery, an intraoperative saliva sample was collected. At the end of surgery after suturing, the oral cavity was irrigated with normal saline and the final saliva sample was collected. The samples were collected using Cocoro meter chips (Nipro) by placing the strip on the floor of the mouth under the tongue for 15 seconds until it was soaked with sa-

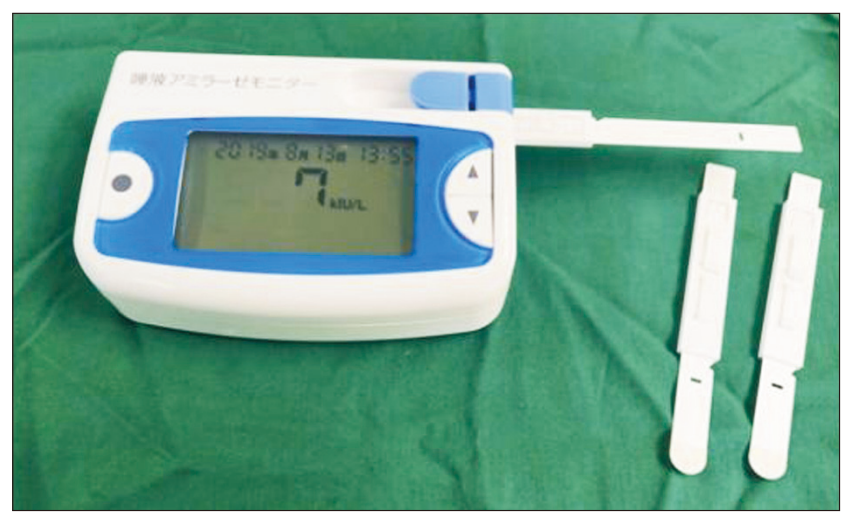

Fig. 1. Cocoro salivary alpha amylase biosensor (Nipro, Japan). Vibha Vaswani et al: Comparative evaluation of salivary alpha amylase level for assessment of stress during third molar surgery with and without piano music and co-relation with pain catastrophizing scale: an in vivo study. J Korean Assoc Oral Maxillofac Surg 2020 
liva and then was mounted on the biosensor. Readings for the concentration of sAA were obtained immediately from the biosensor.

After surgical extraction, patients were asked to complete a self-report of the anxiety experienced during surgery using the PCS. Those readings were tested for correlation with the PCS completed before the surgery. At two weeks after surgery, the procedure was repeated on the other side (right/left).

\section{Statistical analyses}

Data obtained were compiled in a Microsoft Excel 2010 (Microsoft, Redmond, WA, USA) and subjected to statistical analysis using IBM SPSS Statistics (ver. 22.0; IBM, Armonk, NY, USA). The Wilcoxon sign rank test also was used for assessment.

\section{Results}

Comparison of the levels of sAA on the right and left sides with music per random allocation showed lower postoperative level of sAA in patients who listened to piano music before and during surgery compared to preoperative values. Patients who underwent surgery on the right side with music exhibited statistical significance $(P=0.046)$.(Table 1)

Comparison of pain experience using the PCS value preoperatively and postoperatively with music during surgical removal of third molars on either the left or right side exhibited statistically significant results $(P=0.038)$ on the right side, indicating that the catastrophizing reported by the patient before surgery was greater than after surgery.(Table 2)

It was also noted that there was a gradual increase in sAA level intraoperatively in the non-music setup, with a mean sAA level of $46 \mathrm{KU} / \mathrm{L}$, whereas the mean intraoperative sAA level in the music setup was $38 \mathrm{KU} / \mathrm{L}$.

Table 1. With music: preoperative and postoperative comparisons of mean salivary alpha amylase (SAA) level on the two sides

\begin{tabular}{lcc}
\hline \multicolumn{1}{c}{ Side } & $\begin{array}{c}\text { Mean sAA level with } \\
\text { music (KU/L) }\end{array}$ & $P$-value \\
\hline Right & 52 & 0.046 \\
Preoperative & 10 & \\
Postoperative & & 0.109 \\
Left & 45 & \\
Preoperative & 28 & \\
Postoperative & & \\
\hline
\end{tabular}

Vibha Vaswani et al: Comparative evaluation of salivary alpha amylase level for assessment of stress during third molar surgery with and without piano music and co-relation with pain catastrophizing scale: an in vivo study. J Korean Assoc Oral Maxillofac Surg 2020
No statistical significance was found between sAA level and patient self-reported PCS.

\section{Discussion}

An individual's concern regarding his/her physical wellbeing, the known fact that he/she has to be admitted to a hospital or has to undergo a minor out-patient procedure that is anticipated as an unpleasantly noxious one, and worrying about recovery and other pertaining factors make surgery a very baleful experience ${ }^{2}$. As maxillofacial surgeons, we conduct most procedures under local anesthesia, with surgical extraction of impacted mandibular third molars performed routinely. Even though it is a minor oral surgical procedure, it can have a deleterious effect on a patient's emotional and mental well-being.

Anxiety and emotional distress affect postoperative recovery to a great extent considering an individual's personal behavior regarding fear of pain and unpleasant experiences.

Catastrophizing means "to view or present a situation as considerably worse than it actually is." Therefore, such behavioral responses to pain can be attributed to an individual catastrophizing the experience prior to the scheduled surgery. The PCS was developed by Sullivan et al. ${ }^{11}$ in 1995 and is now used in many fields of clinical research to understand an individual's personality traits and to make the patient's surgical experience a comfortable one by assessing his/her response toward pain and preoperative anxiety. This scale is a questionnaire that evaluates catastrophizing using three measurements - rumination, exaggeration, and helplessness ${ }^{11}$.

sAA is released in saliva as a physiologic process, but it is stimulated by the autonomic nervous system, also referred to as the adrenergic system. It was suggested by Gilman et al. ${ }^{12}$ in 1979 that sAA can be used as a biomarker to evaluate stress $^{7}$ through its correlation with increase in psychological

Table 2. With music: preoperative and postoperative comparisons of mean PCS values on the two sides

\begin{tabular}{lcc}
\hline Side & $\begin{array}{c}\text { Mean PCS value with } \\
\text { music (KU/L) }\end{array}$ & $P$-value \\
\hline Right & 25 & 0.038 \\
Preoperative & 8 & \\
Postoperative & & 0.109 \\
Left & 26 & \\
Preoperative & 4 & \\
Postoperative & & \\
\hline
\end{tabular}

Vibha Vaswani et al: Comparative evaluation of salivary alpha amylase level for assessment of stress during third molar surgery with and without piano music and co-relation with pain catastrophizing scale: an in vivo study. J Korean Assoc Oral Maxillofac Surg 2020 
stress. A series of clinical experiments and research was performed to compare the levels of sAA when an individual was subjected to a stressful activity - for example, students appearing for exams and those undergoing relaxation. Over the years, research has reached a level where one can now use a hand-held machine such as a biosensor and disposable strips to determine the value of sAA. The normal resting level of sAA is $38 \mathrm{U} / \mathrm{mL}$ (range, 3.09-48.07 U/mL) and the normal stimulated level of sAA is $38 \mathrm{U} / \mathrm{mL}$ (range, 5.48-56.50 U/ $\mathrm{mL})^{13}$.

Since classical music tends to have a soothing effect on individuals, a pre-recorded classical form of piano music was chosen for this study. There have been many studies published supporting the correlation of psychologic stress reduction and music. In 2008, Camara et al. ${ }^{14}$ chose semiclassical and classical genres of piano music as a relaxation technique during minor ophthalmic surgeries.

To make the surgical experience for the patient comfortable, it is essential to consider all factors including a patient's preoperative perception of pain and fear of an unpleasant experience ${ }^{1}$. Keeping in mind that sAA is a non-invasive and reliable biomarker for estimation of psychological stress and can be evaluated with biosensors, this study was conducted to evaluate the levels of sAA prior to surgical extraction of third molars as well as postoperatively by exposing patients to pre-recorded classical piano music with headphones for approximately 45 minutes (duration of the surgical procedure) to determine its potential influence in reduction of surgical stress. The levels of sAA were compared when a patient was and was not exposed to music.

The diurnal variation in sAA has been studied by Nater et al. ${ }^{15}$, who concluded that level of sAA was lower immediately upon awakening and gradually increased during the course of the day and was greatly affected by stress-inducing factors.

In our study, patients were divided into two main groupsthe music and non-music groups. The side of extraction with or without music was determined randomly. Evaluation of sAA at all given times revealed significant postoperative reduction compared to preoperative values when extraction was performed with music. Among the seven patients, four underwent extraction on the right side with music, while three did not listen to music. Similarly, for the left side, three patients underwent extraction with music, while four did not receive music.

Extractions of the right side with a music set up had a mean preoperative value of SAA at $52 \mathrm{KU} / \mathrm{L}$ and a postoperative value of $10 \mathrm{KU} / \mathrm{L}$, showing a significant difference $(P=0.046)$.
Pain experience was also assessed on the basis of the selfreported PCS where music was played while the procedure was performed on the right side. The mean value of PCS preoperatively was $25 \mathrm{KU} / \mathrm{L}$ and that postoperatively was $8 \mathrm{KU} / \mathrm{L}$. The observed statistical significance $(P=0.038)$ indicates that patients reported more catastrophizing before the procedure compared to after.

In the non-music set up, those who underwent extraction on the left side exhibited statistically significant results, with the PCS reported preoperatively (30.5) being much greater than the postoperative value (20.8) $(P=0.031)$.

We also wanted to correlate sAA with patient self-reported PCS, but no statistical significance was reported in either the music or non-music setup.

Therefore, we conclude that there were statistical differences in SAA level and self-reported pre and postoperative PCS when extractions were performed with pre-recorded classical piano music in the operatory, and no statistical difference was noted between sAA level and self-reported PCS.

\section{Conclusion}

In our study, we evaluated sAA level in patients undergoing surgery for removal of impacted mandibular third molars. The test patients were subjected to pre-recorded classical piano music in the operatory during surgery, and the level of sAA was measured at three intervals and tested for correlation with patient self-reported PCS. Statistically significant reduction of sAA level was were observed in patients who underwent surgical extraction with music in the operatory compared to those who did not listen to music, while statistical significance was not observed in correlation of sAA level and PCS. As a result, we concluded that SAA level exhibits a significant reduction in the presence of music. An additional long-term, prospective, randomized controlled trial must be undertaken to compare the levels of sAA to assess surgeryrelated stress and possible beneficial effects of music.

Our study lacked adequate measurement of baseline sAA activity before patient arrival at the clinic, such as its measurement at the same time on a different day. Our results were limited to the day of surgery, which may be a confounding factor influencing the level of sAA during the procedure. Our study constitutes a small sample size, and further larger studies must be performed.

With the easy availability of the point of care biosensor system the Cocoro meter, surgeons can measure and analyze the patterns of biomarkers like sAA. They will also be able 
to anticipate pain control and determine steps to improve the intraoperative and postoperative care of patients such as individualized anti-anxiety strategies and various medications for pain.

\section{ORCID}

Vibha Vaswani, https://orcid.org/0000-0002-7504-7271

Sonal Shah, https://orcid.org/0000-0001-8424-0105

Manne Lakshmipriyanka, https://orcid.org/0000-0003-

1552-7692

Pushkar Waknis, https://orcid.org/0000-0002-8230-6075

Deeisha Gupta, https://orcid.org/0000-0003-3044-7084

Kunal Jain, https://orcid.org/0000-0002-7921-7362

\section{Author's Contributions}

V.V. participated in data collection and wrote the manuscript. S.S. and P.W. participated in the study design and performed the statistical analysis. M.L., D.G., and K.J. participated in the study design and coordination and helped to draft the manuscript. All authors read and approved the final manuscript.

\section{Ethics Approval and Consent to Participate}

This study was conducted after obtaining approval from the Institutional Ethics Committee of Dr. D. Y. Patil Dental College \& Hospital, Pune (DYPDCH/IEC/1262/14118), and written informed consent was obtained from all participants.

\section{Conflict of Interest}

No potential conflict of interest relevant to this article was reported.

\section{References}

1. Robles TF, Sharma R, Park KS, Harrell L, Yamaguchi M, Shetty V. Utility of a salivary biosensor for objective assessment of surgeryrelated stress. J Oral Maxillofac Surg 2012;70:2256-63. https://doi. org/10.1016/j.joms.2012.03.005

2. Kiecolt-Glaser JK, Page GG, Marucha PT, MacCallum RC, Glaser R. Psychological influences on surgical recovery. Perspectives from psychoneuroimmunology. Am Psychol 1998;53:1209-18. https://doi.org/10.1037/0003-066X.53.11.1209

3. Edwards RR, Fillingim RB, Maixner W, Sigurdsson A, Haythornthwaite J. Catastrophizing predicts changes in thermal pain responses after resolution of acute dental pain. J Pain 2004;5:164-70. https://doi.org/10.1016/j.jpain.2004.02.226

4. Sullivan MJ, Thorn B, Haythornthwaite JA, Keefe F, Martin M, Bradley LA, et al. Theoretical perspectives on the relation between catastrophizing and pain. Clin J Pain 2001;17:52-64. https://doi. org/10.1097/00002508-200103000-00008

5. Sullivan MJ, Neish NR. Catastrophizing, anxiety and pain during dental hygiene treatment. Community Dent Oral Epidemiol 1998;26:344-9. https://doi.org/10.1111/j.1600-0528.1998.tb01971.x

6. Nater UM, Rohleder N, Gaab J, Berger S, Jud A, Kirschbaum C, et al. Human salivary alpha-amylase reactivity in a psychosocial stress paradigm. Int J Psychophysiol 2005;55:333-42. https://doi. org/10.1016/j.ijpsycho.2004.09.009

7. Nater UM, Rohleder N. Salivary alpha-amylase as a non-invasive biomarker for the sympathetic nervous system: current state of research. Psychoneuroendocrinology 2009;34:486-96. https://doi. org/10.1016/j.psyneuen.2009.01.014

8. Bosch JA, Veerman EC, de Geus EJ, Proctor GB. $\alpha$-Amylase as a reliable and convenient measure of sympathetic activity: don't start salivating just yet! Psychoneuroendocrinology 2011;36:449-53. https://doi.org/10.1016/j.psyneuen.2010.12.019

9. Shetty V, Zigler C, Robles TF, Elashoff D, Yamaguchi M. Developmental validation of a point-of-care, salivary $\alpha$-amylase biosensor. Psychoneuroendocrinology 2011;36:193-9. https://doi.org/10.1016/ j.psyneuen.2010.07.008

10. Wang SM, Kulkarni L, Dolev J, Kain ZN. Music and preoperative anxiety: a randomized, controlled study. Anesth Analg 2002;94:1489-94. https://doi.org/10.1097/00000539-20020600000021

11. Sullivan MJL, Bishop SR, Pivik J. The pain catastrophizing scale: development and validation. Psychol Assess 1995;7:524-32. https:// doi.org/10.1037/1040-3590.7.4.524

12. Gilman S, Thornton R, Miller D, Biersner R. Effects of exercise stress on parotid gland secretion. Horm Metab Res 1979;11:454. https://doi.org/10.1055/s-0028-1095789

13. Arhakis A, Karagiannis V, Kalfas S. Salivary alpha-amylase activity and salivary flow rate in young adults. Open Dent J 2013;7:715. https://doi.org/10.2174/1874210601307010007

14. Camara JG, Ruszkowski JM, Worak SR. The effect of live classical piano music on the vital signs of patients undergoing ophthalmic surgery. Medscape J Med 2008;10:149.

15. Nater UM, Rohleder N, Schlotz W, Ehlert U, Kirschbaum C. Determinants of the diurnal course of salivary alpha-amylase. Psychoneuroendocrinology 2007;32:392-401. https://doi.org/10.1016/ j.psyneuen.2007.02.007

How to cite this article: Vaswani V, Shah S, Lakshmipriyanka M, Waknis P, Gupta D, Jain K. Comparative evaluation of salivary alpha amylase level for assessment of stress during third molar surgery with and without piano music and co-relation with pain catastrophizing scale: an in vivo study. J Korean Assoc Oral Maxillofac Surg 2020;46:235-239. https://doi.org/10.5125/jkaoms.2020.46.4.235 